\title{
Étude comparée des interrogateurs en lama et en français
}

\author{
Hanoukoume Cyril Kparou, (PhD)
}

Babcock University, School of Education and Humanities, Department of Languages and Literary Studies, Ogun State, Nigeria

Doi:10.19044/esj.2018.v14n26p332 URL:http://dx.doi.org/10.19044/esj.2018.v14n26p332

\begin{abstract}
Interrogation is a Language Universal (LU), i.e., it exists in all natural languages. However, every language has undeniable particularities. Lama ${ }^{3}$ and French ${ }^{4}$, two languages from different language families, are a good illustration of these realities. A comparative study of interrogatives of both languages shows many similarities as well as dissimilarities. This article is aimed at presenting a comparative typology of interrogative markers and constructions in Lama and French. It consists of a morphosyntactic analysis of the interrogative markers and their different functions implied in the construction of interrogative sentences. French uses an interrogative copula est-ce que and the verbal inversion to form total interrogation, while Lama makes use of postpositional morphemes to formulate fundamental interrogation. Both languages form partial interrogation with adverbial and relative markers, but Lama goes further with the use of noun class interrogative markers. This study contributes to establish language universals and particularities in both languages at formal and functional levels.
\end{abstract}

Keywords: Interrogation, total interrogation, partial interrogation, Lama, French.

\section{Résumé}

La ' 'question' est un universaux du langage, c'est-à-dire, il n'existe point de langue sans constructions interrogatives. Le lama et le français, deux langues issues de familles linguistiques différentes, illustrent bien ces réalités. La comparaison des deux langues fait ressortir à la fois des points de divergence et des similitudes mineures. Le présent article a donc pour objectif

${ }^{3}$ Lama (ISO 639-3 las): Niger-Congo, Atlantic-Congo, Volta-Congo, North, Gur, Central, Southern, Gurunsi, Eastern, Lama (source: www.ethnologue.com).

${ }^{4}$ French (ISO 639-3 fra): Indo-European, Italic, Romance, Italo-Western, Western, GalloIberian, Gallo-Romance, Gallo-Rhaetian, Oil, French (source: www.ethnologue.com). 
de présenter une typologie comparative des constructions interrogatives du lama et du français. Ceci passe par l'analyse morphosyntaxique des morphèmes interrogateurs et l'étude des différentes dérivations impliquées dans les constructions interrogatives. Alors que le français utilise la copule interrogative « est-ce que » et l'inversion ou non du sujet pour formuler l'interrogation, le lama utilise les morphèmes postposés au verbe pour formuler l'interrogation totale fondamentale et l'interrogation d'acquiescement. On remarque la présence dans les deux langues d'interrogateurs adverbiaux et relatifs, mais le lama se distingue à ce niveau par la présence d'interrogateurs prototypes de genres. Cette étude contribue à établir d'une part, le concept d'universaux du langage, et d'autre part, la singularité linguistique à la fois formelle et fonctionnelle.

Mots-clés : Interrogation, interrogation totale, interrogation partielle, lama, français.

\section{Introduction :}

L'interrogation est une modalité linguistique de formulation de questions. Selon Crystal (2008), et Choi-Jonin \& Delhaye (1998), la présence d'une interrogation exprime un souci de renseignement de la part du locuteur, et pour Leeman (2002), elle exige une responsabilité de réponse ou de justification de la part de l'interlocuteur.

Toutefois, toute question n'exprime pas forcément un souci de renseignement, encore moins une réponse. La question «c'est à moi tout ceci ? » posée lors de la réception d'un cadeau n'exprime aucun souci de renseignement, mais plutôt une surprise. La question de Pharaon (Sainte Bible, Exode 5:2) « qui est l'Éternel pour que j'obéisse à sa voix, en laissant aller Israël ? » n'était qu'un défi et non un souci de renseignement de la part du monarque.

Il existe dans les langues du monde, diverses formes interrogatives qui ont pu être regroupées en quatre catégories par souci méthodologique. Il existe d'une part, les questions directes opposées à celles qui sont dites indirectes et les questions totales qui sont en dichotomie avec leurs correspondantes partielles (Choi-Jonin \& Delhaye, 1998; Tellier, 2003).

Bien qu'elle soit admise comme telle en grammaire normative, cette distinction ne fait pas l'unanimité, car, elle n'est pas distributionnelle, à tel point que les phrases analysées dans la première dichotomie se retrouvent dans la seconde. C'est pour tenter de palier à ce problème que Strik (2008) propose un autre regroupement. Ce dernier auteur distingue en général une dichotomie: les questions totales et les questions partielles dites aussi questions "'wh". C'est au sein des questions ' wh' qu'on distingue les questions wh - simples, les questions wh - longues distance et les questions wh - indirectes. C'est sur 
le modèle de Strik que nous structurons les constructions interrogatives en lama, toutefois, avec une différence de fond.

\section{L'interrogation totale:}

L'interrogation est totale quand elle est basée sur une valeur de vérité. Elle est entièrement articulée autour du contenu propositionnel permettant à l'interlocuteur d'asserter par oui ou non. Strik (2008) soutient à juste titre que la question dite « totale » porte sur l'entièreté de l'interrogation.

En français, il y a fondamentalement trois mécanismes de formulation d'une interrogation totale. Il y a d'abord, les phrases interrogatives introduites par le morphème dislocateur « est-ce que » (1), ensuite, les formes interrogatives marquées par l'inversion du sujet (2), et enfin, l'interrogation totale sans inversion du sujet (3). Cette dernière forme ressort plus ou moins du style familier.

Toutefois, on peut ajouter à ces trois formes, l'interrogation alternative, comme l'illustre l'exemple (4).

(1) Est-ce que vous mangez du poisson ?

(2) Mangez-vous du poisson ?

(3) Vous mangez du poisson ?

(4) Mangez-vous du poisson ou du poulet?

En lama par contre, on distingue au sein de l'interrogation totale une forme fondamentale avec des morphèmes réguliers, l'interrogation d'acquiescement semblable au 'question tag"' anglais et l'interrogation alternative.

Une différence majeure à noter est que, contrairement au français, les morphèmes interrogateurs en lama sont postposés.

\subsection{L'interrogation totale fondamentale}

Pour l'interrogation totale fondamentale, les morphèmes yá, káá, kéć, ná, níl et néć, se partagent les contextes.

(5)

a. Alika tówá yá ?

/Alika/manger ACP/INTER/

« Est-ce que Alika a mangé ? »

Le locuteur veut se renseigne objectivement, sans présupposition.

b. Alika tówá káá ?

/Alika/manger ACP/INTER/

« Alika a-t-il mangé ?»

Il se renseigne avec présupposition qu'Alika ou du moins quelqu'un d'autre ait pu manger la chose. Si Alika n'a pas mangé, qui a dû manger ?

c. mín rəmpá kéć ?

/feu/éteindre ACP/INTER/

« Le feu est-il éteint?» 
kéć et káá sont des variantes, mais kéć est de nature péjorative et sert à interroger sur des instances propositionnelles que le locuteur sous-estime comme (d) ; dans ce propos, le locuteur laisse entendre qu'il sous-estimait Alika, il ne s'attendait pas à sa réussite et qu'il en parle avec malaise.

d. Alika tówa kéć ?

/Alika/réussir ACP/INTER/

«Alika (aussi) a réussi ?»

Les interrogateurs yá (a et f) et ná (e) sont en distribution complémentaire avec une objectivité similaire. Le premier s'utilise dans un environnement verbal monoargumental (DP + V + INTER), tandis que le second, ná, apparait dans un contexte biargumental (DP + V + DP + INTER).

e. n lí lém ná ?

/2s/puiser HYP/eau/INTER/

«As-tu puisé de l'eau?»

f. n liwá yá ?

/2s/puiser ACP/INTER/

«As-tu puisé (de l'eau) ?»

Le morphème néć est l'équivalent présupposé et péjoratif de ná. Il exige un contexte biargumental et porte sur des instances propositionnelles que le locuteur sous-estime $(\mathrm{g})$.

g. n tókú túná néź ?

/2s/manger INACP/poisson PL/INTER/

« Mangerais-tu du poisson ? (Je m'étonne que tu manges du poisson !)

níl est peu objectif. Il présuppose une réponse négative.

h. n tókú túná núí?

/2s/manger INACP/poisson PL/INTER/

"Mangerais-tu du poisson? ? (Je sais déjà que tu ne manges pas du poisson!)

Les morphèmes de l'interrogation totale peuvent être dans une dichotomie syntaxique binaire de trois éléments. D'un côté, il y a des interrogateurs qui s'utilisent en contexte verbal monoargumental (DP + V + INTER). C'est le triplet yá, káá et kéć. De l'autre côté, il y a ceux qui requièrent une distribution biargumentale (DP $+\mathrm{V}+\mathrm{DP}+\mathrm{INTER})$. Ces derniers regroupent ná, núí et néć. Dans chaque groupe, il y a deux extrêmes et un intermédiaire. La première extrémité concerne ceux qui s'utilisent en toute objectivité. Il s'agit de yá et ná. La seconde extrémité concerne kéć et néź qui sont à la fois péjoratifs avec une présupposition négative. Les intermédiaires entre les deux extrémités sont káá et níí.

L'ensemble de ces morphèmes peut être résumé dans le tableau suivant : 
Tab. 1 : Tableau de distribution des interrogateurs totaux en lama

\begin{tabular}{|c|c|c|c|}
\hline Distribution syntaxique & $\begin{array}{l}\text { Extrémité } \\
\text { objective }\end{array}$ & Intermédiaire & Extrémité péjorative \\
\hline $\mathrm{DP}+\mathrm{V}+\mathrm{INTER}$ & yá & káá & kéć \\
\hline $\mathrm{DP}+\mathrm{V}+\mathrm{DP}+\mathrm{INTER}$ & ná & núi & néć \\
\hline
\end{tabular}

\subsection{Le "question tag" ou interrogation d'acquiescement}

L'interrogation totale est aussi produite avec le morphème /'y qui se suffixe au dernier mot de la phrase. Il est destiné à renforcer l'acquiescement et correspond à l'incise française «n'est-ce pas ?» Il peut apparaitre seul ou cohabiter avec les interrogateurs ná et yá. Dans les données suivantes, /'ill seconde ná en (a) avec lequel il se fusionne pour former un seul morphème /ńl/ en contexte distributionnel V + DP + INTER. Il seconde yá en (b) dans l'environnement V + INTER.

(6)

a. n tókú túná ní/ n tókú túná na-í ?

/2s/manger INACP/INTER/

« Tu manges du poisson, n'est-ce pas?»

b. n tókú yá-í ?/ n tókú-i ?

/2s/manger INACP/INTER/

« Tu manges, n'est-ce pas ?»

c. n wáló-í ?

/2s/aller INACP - INTER/

« Tu (y) vas, n'est-ce pas?»

d. moná-í ?

/1s FOC - INTER/

«C'est donc moi ?»

\subsection{Les questions alternatives}

L'interrogation alternative est celle qui laisse l'interlocuteur devant un choix pour donner une réponse. Même si dans ce cas l'interlocuteur ne répond pas par oui ou non, et donc, susceptible d'être classée dans l'interrogation partielle, l'interrogation alternative est fondamentalement totale sur trois points principaux :

(i) Elle utilise des morphèmes requis pour l'interrogation totale ;

(ii) La distribution de ces morphèmes est conforme à celle de l'interrogation totale ;

(iii) Elle délimite le cadre de la réponse en proposant au locuteur un choix à opérer. Tout comme l'interrogation totale fondamentale ou le locuteur s'attend à soit oui, ou soit non, le locuteur de l'interrogation alternative s'attend à une réponse parmi les propos introduits par lui-même. 
(7)

a. Alika tówá yá í ñéwá ?

/Alika/manger ACP/INTER/3s/boire ACP/

«Alika a mangé ou il a bu ?»

b. $\mathrm{n}$ lí lém ná $\mathrm{n}$ hásó tón ?

/2s/puiser HYP/eau/INTER/2s/balayer HYP/cour/

« Tu as puisé de l'eau ou balayé la cour?»

c. Alika tú máán ná mótó ?

/Alika/manger ACP/riz/INTER/pâte/

« Alika a mangé du riz ou de la pâte ?»

d. ña ná $n$ kó li ná lém ?

/2s/INTER/Poss 2s/sœur/puiser ACP/FOC/

«C'est toi ou ta sœur qui a puisé de l'eau ?»

e. Alika yá Karka tó ná ?

/Alika/manger ACP/INTER/3s/boire ACP/FOC/

« C'est Alika ou Karka qui a mangé ? »

Les questions alternatives peuvent être analysées en prenant en compte l'objet de l'alternance. Les éléments proposés en alternance peuvent être sujet ou objet de $\mathrm{V}$ ou encore, l'alternance peut concerner des procès verbaux. Dans les exemples ci-dessus, (e) et (d) illustrent le cas d'une question portant sur des éléments en alternance dans le sujet. (c) par contre concerne l'objet, tandis que (a) et (b) concernent des propositions verbales.

Dans ces exemples en lama, il parait évident que les deux interrogateurs fondamentaux de l'interrogation totale se partagent les environnements. L'interrogateur ná n'apparait que lorsqu'il y a un DP dans son contexte précédent (cf (b) (c) et (d)). Dans ces exemples, les interrogateurs sont en gras; à ne pas confondre ná (l'interrogateur) et ná (le focalisateur) ; le deuxième ná en (d), et le ná final en (e) sont des focalisateurs.

La sous-section suivante aborde l'interrogation partielle et ses composantes.

\section{Interrogation partielle}

L'interrogation partielle est celle qui exige de l'interlocuteur une réponse propositionnelle. On distingue deux grands groupes d'interrogation partielle.

Elle est aussi appelée interrogation "wh" à cause de la présence de "wh", dans la plupart des interrogateurs anglais que Chomsky (1977) a étudiés, tels que why, where, who, whose... En français, certains auteurs parlent plutôt des interrogateurs Q ou Qu (Defrancq, 2005) en rapport aux mots de ce type tels que quand, quoi, qui, quel ...

L'interrogation dite partielle est marquée morphologiquement en lama par un morphème. Le suffixe /-o/ (ou sa variante [-ATR], -o) est le moteur principal de questionnement, requis sur tous les mots dits "wh', quelque soit 
leur nature. Nous appelons ce type "'interrogation O' d'après le modèle "wh', de Chomsky (1977).

Plusieurs morphèmes s'associent au morphème /-o/ pour permettre la formulation des questions suivant diverses formes constructionnelles. On distingue les interrogateurs adverbiaux ou circonstanciels, les interrogateurs relatifs et les prototypes des genres.

\subsection{Les interrogateurs adverbiaux}

Ils permettent de poser des questions pour se renseigner sur le temps, le lieu, la cause, la quantité et la manière. Le tableau suivant présente les interrogateurs adverbiaux en lama et leurs correspondants en français.

Tab. 2 : Interrogateurs adverbiaux en lama et en français

\begin{tabular}{|c|c|c|c|c|}
\hline Fonctions & $\begin{array}{l}\text { Interrogateurs } \\
\text { en lama }\end{array}$ & $\begin{array}{l}\text { Traits } \\
\text { morphosyntaxiques }\end{array}$ & $\begin{array}{l}\text { Rôle } \\
\text { syntaxique } \\
\text { in situ }\end{array}$ & $\begin{array}{l}\text { Interrogateurs } \\
\text { en lama }\end{array}$ \\
\hline Temps & uyó & [+Temporel] & \multirow{4}{*}{$\begin{array}{l}\text { Adjoint de } \\
\mathrm{V}\end{array}$} & Quand? \\
\hline Localisation & 10 & [+Locatif] & & Où? \\
\hline Cause & səmo & [+Causatif] & & Pourquoi? \\
\hline Manière & sənó & [+Manière] & & Comment? \\
\hline Quantité & ḿmó & [+Quantitatif] & $\begin{array}{l}\text { Sujet, } \\
\text { Objet, } \\
\text { Adjoint }\end{array}$ & Combien? \\
\hline
\end{tabular}

La position in situ des interrogateurs adverbiaux est en final de la proposition (9). Il y a cependant, des formes ex situ avec les éléments $\mathrm{O}$ à l'initial (10).

(8)

a. Alika kám uyó ?

/Alika/venir HYP/quand INTER/

«Alika est venu quand?»

b. n wálú lo ?

/2s/aller INACP/où INTER/

«Où vas-tu?»

c. yó kó səm? ?

/enfant/pleurer INACP/pourquoi INTER/

« Pourquoi l'enfant pleure-t-il ?»

d. a hárú sənó ?

/IndP/cultiver INACP/comment INTER/

«Comment cultive-t-on?»

e. yír nín we múmó ?

/personne/main/être COP/ combien INTER/

«Combien de mains l'homme possède-t-il ?»

(9)

a. uyó Alika kámpá ? 
/quand INTER/Alika/venir ACP /

« Alika est venu quand?»

b. lo n wálú ?

/où INTER /2s/aller INACP /

«Où vas-tu?»

c. səmつ yó kó ?

/pourquoi INTER /enfant/pleurer INACP/

« Pourquoi l'enfant pleure-t-il ? »

d. sənó a hárú ?

/comment INTER/IndP/cultiver INACP/

« Comment cultive-t-on?»

e. ḿmó kle'?

/Combien INTER /DEM/

«C'est combien?»

\subsection{Les interrogateurs relatifs}

Les pronoms interrogateurs sont de deux types. On distingue les indéfinis et les prototypes de genres en lama.

\subsubsection{Les interrogateurs indéfinis}

Les indéfinis servent à poser une question au sujet des choses et des personnes indéterminées. On peut les classer dans le tableau suivant :

Tab. 3 : Tableau des interrogateurs relatifs en lama et en français

\begin{tabular}{|c|c|c|c|c|c|}
\hline & $\begin{array}{l}\text { Singulie } \\
\mathbf{r}\end{array}$ & Pluriel & $\begin{array}{l}\text { Traits } \\
\text { morphosyntaxiques }\end{array}$ & $\begin{array}{l}\text { Rôle } \\
\text { syntaxique } \\
\text { in situ }\end{array}$ & $\begin{array}{l}\text { équivalents } \\
\text { français }\end{array}$ \\
\hline $\begin{array}{l}\text { Indéfinis } \\
\text { personnels }\end{array}$ & ánó & ámpós & [+Pers] & $\begin{array}{l}\text { Sujet ; } \\
\text { Objet }\end{array}$ & Qui ? \\
\hline \multirow{3}{*}{$\begin{array}{l}\text { Indéfinis } \\
\text { impersonnels }\end{array}$} & wo & & [-Pers] & Objet & Quoi? \\
\hline & átı & átıwo & [-Pers, + Dimin] & Objet & Quoi? \\
\hline & ár & & [-Pers] & $\begin{array}{l}\text { Sujet ; } \\
\text { Objet }\end{array}$ & Quoi? \\
\hline
\end{tabular}

NB : Pers : personnel ; Dimin : diminution

(10)

a. 1 wósú ár $\mathrm{r} \varepsilon$

/3s/demander INACP/INTER/sur/

«Il demande d'après quoi ? »

b. * 1 wósú ár

c. 1 wósú wo ?

/3s/demander INACP/INTER/

«Il demande quoi ?»

d. wo í wósú ? 
/INTER/3s/demander INACP /

«Il demande quoi ? »

e. ámpó kampá ?

/qui PL/venir ACP/INTER/

«Qui sont ceux qui sont venus?»

f. n yáá áno ?

/2s/appeler ACP/qui SG/INTER/

«Qui appelles-tu?»

g. 1 lákó átı ?

/3s/faire INACP/INTER/

«il fait quoi ?»

h. átı í lákó ?

/INTER/3s/faire INACP/

«que fait-il ?»

i. ??átı lapá ? [style très familier]

/INTER/faire ACP/

«Que s'est-il passé ? »

j. ár lapá ?

/INTER/faire ACP/

«Que s'est-il passé ?»

Les O [+Pers] ont pour équivalents « qui ? ». Ils ont des positions in situ aussi bien à l'initial qu'en final. En (e), l'élément $\mathrm{O}$ est à l'initial et sujet du verbe. En (f), la phrase clôt avec le mot $\mathrm{O}$ en tant que complément interne du verbe.

Les indéfinis wo, átı et ár occupent des positions distributionnelles in situ. La position principale de wo « quoi ? » est en final en rôle syntaxique d'objet (c). L'exemple (d) illustre la position ex situ du même élément, en conservant toutefois le rôle syntaxique d'objet de $\mathrm{V}$.

L’indéfini átı « quoi ? », est de nature péjorative. Sa position standard est en final de la proposition avec le rôle syntaxique d'objet (g). Il peut toutefois être à l'initial (h) en conservant son rôle syntaxique d'objet. Des expressions avec cet interrogateur en rôle syntaxique de sujet sont du registre familier (i).

L'interrogateur ár « quoi ? » est hostile à la position finale, ce qui explique l'agrammaticalité de (b). Cette exclusion explique une réalité : ár n'occupe pas la position d'objet direct de V. il peut être sujet et donc, à l'initial comme (j) ; ou complément PP (accompagné d'une postposition) comme (a).

Les interrogateurs indéfinis s'opposent aux interrogateurs définis par leur appartenance aux genres.

\subsubsection{Interrogateurs prototypes de genres}

Ils servent à poser des questions en rapport avec des éléments selon le genre et le nombre. En lama, ils sont formés des anaphores discursives 
suffixées au morphème interrogateur selon la règle morphologique cidessous :

(11) $\quad$ INTER $\rightarrow$ ANAPH $+\mathrm{O}$

L'ensemble des interrogateurs prototypes de genres se présente dans le tableau suivant :

Tab. 3 : Interrogateurs prototypes de genres

\begin{tabular}{|c|c|c|c|}
\hline & Singulier & Pluriel & $\begin{array}{l}\text { Équivalents } \\
\text { français }\end{array}$ \\
\hline G1 & (wó)woñó & wompó & \multirow{6}{*}{$\begin{array}{l}\text { Quel/quelle ; } \\
\text { quels/quelles ; } \\
\text { Lequel/laquelle ; } \\
\text { Lesquels/lesquelles } \\
\text { Quoi + Genre. }\end{array}$} \\
\hline $\mathrm{G} 2$ & wónró & wónyó & \\
\hline G3 & wónkó & wónsó & \\
\hline G4 & \multicolumn{2}{|l|}{ wóntó } & \\
\hline G5 & \multicolumn{2}{|l|}{ wómpó } & \\
\hline G6 & wónkó & wónñó & \\
\hline
\end{tabular}

Les interrogateurs prototypes de genres sont du domaine de l'anaphore phrastique, c'est-à-dire, pouvant apparaitre avec leurs antécédents dans la même proposition.

(12)

a. yó wónká kampá ?

/enfant/INTER/venir ACP/

«Quel enfant est venu ?»

b. n yáá yó wónkj ?

/2s/appeler HYP/enfant/INTER/

«Quel enfant tu as appelé ? »

c. Wísá nasól kampá. n tórú wonkó ?

/enfant PL/deux G3/venir ACP/. /2s/envoyer INACP/INTER/

«Deux enfants sont venus. Lequel tu veux envoyer?»

d. n wótú wóntó ?

/2s/dire INACP/quoi G4 INTER/

« Tu dis quoi (quelle parole) ? »

Dans les données ci-dessus, (a) montre l'interrogateur dans un environnement phrastique autre que l'initial. C'est en raison de sa position médiane qu'il est réduit de /-ऽ/. Dans la même position, il partage le rôle syntaxique de sujet avec son antécédent 'yó'. En (b), l'interrogateur est toujours dans un environnement phrastique, mais en final de proposition et partage avec son antécédent le rôle syntaxique le complément interne de $\mathrm{V}$.

Les exemples (c) et (d) sont des cas où l'interrogateur prototype de genre apparait dans un environnement discursif, c'est-à-dire, loin ou en absence de son antécédent. En (c), le contexte précédent contient l'antécédent de l'interrogateur. Ce qui n'est pas le cas en (d) où il n'existe aucun environnement permettant une référence directe. Mais les locuteurs du lama le référèrent à '‘t́m'" «parole », mot qui est du G4 et s'accorde avec l'interrogateur wóntó. 
En définitive, l'analyse des interrogateurs a permis de faire un regroupement de question par type d'élément $\mathrm{O}$, qu'il soit adverbial ou relatif.

\section{Conclusion}

Cette étude a permis d'analyser les interrogateurs et types d'interrogation en lama et en français dans une perspective comparative. On peut admettre que les deux grands types d'interrogation, à savoir l'interrogation totale et l'interrogation partielle, se retrouvent aussi bien en lama qu'en français. Toutefois, les différences sont assez notoires. Alors que l'interrogation totale peut concerner plus ou moins quatre formes en français, elle est réduite à trois formes en lama. Ainsi, le français utilise la copule interrogative "est-ce que » et l'inversion ou non du sujet pour formuler l'interrogation, alors que le lama utilise les morphèmes postposés au verbe pour formuler l'interrogation totale fondamentale et l'interrogation d'acquiescement. Les deux langues font recours à l'interrogation alternative.

Les deux langues forment également l'interrogation partielle. On remarque la présence dans les deux langues d'interrogateurs adverbiaux, d'interrogateurs relatifs, mais le lama se distingue à ce niveau par la présence d'interrogateurs prototypes de genres.

Cette étude contribue à établir d'une part, le concept d'universaux du langage, et d'autre part, la singularité linguistique à la fois formelle et fonctionnelle. Nous recommandons ce travail dans le contexte de la linguistique comparative, et dans l'enseignement de la langue, à la fois comme langue première et langue étrangère.

\section{References:}

1. Adger, D. (2003). Core Syntax: A Minimalist Approach. Oxford: Oxford University Press.

2. Baylon, C. \& Fabre, P. (2007). Initiation à la linguistique. Paris: Armand colin.

3. Choi, J. \& Delhay, C. (1998). Introduction à la méthodologie en linguistique, Application au français contemporain. Strasbourg: PUS.

4. Chomsky, N. (1977). "On Wh-movement". Formal syntax. 71-132. New York: Academic Press.

5. Chomsky, N. (2008). "On Phases". Foundational issues in linguistic theory: essays in honor of Jean-Roger Vergnaud. 133-166. Cambridge, Massachusetts: MIT Press.

6. Creissels, D. (2006). Syntaxe générale, une introduction typologique, v1. Paris : Lavoisier.

7. Crystal, D. (2008). A Dictionary of Linguistics and Phonetics. Sixth Edition, Blackwell Publishing. 
8. Feuillet, J. (2006). Introduction à la typologie linguistique. Paris: Honoré Champion.

9. Fournier, D. H. (2010). La structure du prédicat verbal : une étude de la construction à double objet en français. Ph.D. Dissertation, University of Toronto.

10. Gardes-Tamine, J. (2004). La grammaire : 2. Syntaxe. Paris : Armand Colin.

11. Kayne, S. R. (1977). Syntaxe du Français. Le cycle transformationnel. Paris: Seuil.

12. Leeman, D. (2002). La phrase complexe: les subordinations. Bruxelles: Duculot.

13. Neveu, F. (2000). Lexique des notions linguistiques. Paris: Nathan.

14. Defrancq, B. (2005). L'interrogative enchâssée. Structure et interprétation. Bruxelles: De Boeck.

15. Ourso, M. A. (2013). "Casse-tête chinois pour l'hypothèse de l'intégrité lexical'. Particip 'Action, Revue Interafricaine de littérature, linguistique et philosophie, Université de Lomé, vol5, N02, Janvier 2013, $207-226$.

16. Strik, N. (2008). Syntaxe et acquisition des phrases interrogatives en français et en néerlandais: une étude contrastive. Thèse de Doctorat, Université Paris 8 - Saint Denis.

17. Tellier, C. (2003). Éléments de syntaxe du français, méthodes d'analyse en grammaire générative, $2^{\mathrm{e}}$ edition, Montreal: Gaetan Morin. 